\title{
APERÇU SUR LA PROCÉdure PÉNAle AUSTRALIENNE
}

\section{Bron McKellop*}

\section{Section I : Considérations générales}

\section{§ 1- Introduction}

L'architecture de l'organisation politique en Australie repose sur le principe du fédéralisme. La Constitution Fédérale de 1900 a organisé un partage des pouvoirs législatifs entre d'une part le Parlement Fédéral du "Commonwealth" d'Australie (dont le siège se trouve à Canberra) et les Parlements des six États qui composent l'Australie, d'autre part.

Dans ce cadre institutionnel, le droit pénal échappe par principe à la compétence du Parlement Fédéral et seuls les Etats peuvent légiférer en cette matiere. Toutefois, il ne faut pas en conclure que le Parlement Fédéral soit dépourvu de toutes prérogatives dans ce domaine. En effet le Parlement Fédéral pourra toujours voter des dispositions qui manifestement s'insèrent dans le cadre de ses propres compétences législatives. Ainsi a titre d'exemple, il peut toujours assortir de sanctions pénales les mesures tendant à interdire l'importation des stupéfiants dès lors qu'il s'agit là d'une prérogative qui s'inscrit dans son pouvoir en matiere de commerce extérieur.

En conséquence, les juridictions qui ont à connaître des affaires pénales seront celles de chaque Etats concerné qui auront également une compétence de droit commun pour édicter les règles de procédure qui leur sont propres. Cette compétence s'exerce même pour les affaires pénales relatives à une violation d'une loi fédérale. Dans cette derniere hypothèse les juridictions des Etats exercent une compétence d'exception, à savoir le pouvoir judiciaire du Commonwealth, dont elles sont investies en vertu d'une loi de Commonwealth.

Le droit pénal de trois Etats d'Australie (Nouvelles Galles du Sud, Victoria, South Australia) apparaît comme un mélange de règles écrites et de "Common Law". La traduction exacte de l'expression "Common Law" n'est pas aisée. On peut toutefois dire sans crainte d'etre démenti, qu'il s'agit des règles qui émanent des juridictions plutôt que du Parlement. Tout comme en Angleterre, les principes généraux du droit pénal ont en Australie, valeur de

* Professeur de Droit à la Faculté de Droit, Université de Sydney. 
normes supérieures du droit. Ces principes ont été dégagés au fil des siècles par les tribunaux. C'est ainsi que les principes relatifs à la démence, l'ivresse, l'erreur, la contrainte, et la légitime défense, s'imposent de manière égale et uniforme dans ces trois Etats. Il s'agit là d'un processus continuel qui aujourd'hui incombe aux seules juridictions supérieures de ces états, en dernier ressort à la high Court, et qui tend à la découverte de nouveaux principes. Par contre le support de la qualification pénale des faits reprochés (crimes et délit ou contravention) est de plus en plus du ressort des Parlements de chaque état, les préceptes de la "Common Law" demeurant cependant toujours présents (par exemple pour la définition de la notion de démence).

Les trois autres Etats (Queensland, Western Australia et Tasmania) ont quant à eux des Codes Pénaux qui datent du début de ce siècle. Il reste que ces Codes sont eux aussi basés sur la "Common Law" telle qu'elle était conçue à l'époque de la rédaction de ces codes. De surcroît, l'interprétation de leurs dispositions se fera toujours dans l'esprit de la "Common Law". On notera que ces trois Etats n'ont toutefois pas de Code de Procédure Pénale à proprement parler.

En Australie le procès pénal s'organise autour de l'alternative suivante: avec jury ou sans jury.

Schématiquement, le procès avec jury est réservé pour les crimes et les délits que l'on pourrait qualifier de sérieux (assassinats, meurtres, viols, coups et blessures graves, vols qualifiés par exemple). Ces procès où siègent un juge et douze jurés sont dans l'organisation judiciaire australienne de la compétence exclusive des juridictions supérieures ("Supreme Court", "District Court" en Nouvelle Galles du Sud). composent.

Les procès sans jury, intéressent quant à eux , ce que l'on pourrait qualifier de délits d'importance moindre et les contraventions. Ils sont de la compétence de juridictions inférieures ("Local Court") où siège un juge unique appelé "Magistrate", qui exerce une juridiction qui à bien des égard peut apparaître sommaire comparée à celle que se déroule dans les procès avec jury. En effet si quantitativement, la plupart des procès pénaux ont lieu devant les "Magistrates", qualitativement parlant, dès lors que les procédures y sont moins formelles que devant les juridictions supérieures, le déroulement de ces procès ne qu'un intérêt limité et c'est pourquoi les observations suivantes porteront principalement sur les procès avec jury qui se déroulent en Nouvelle Galles du sud, l'Etat le plus peuplé d'Australie.

\section{§ 2- Les différentes phases du procès du pénal}

Cinq phases peuvent etre considérée. Toutes correspondent à une étape spécifique de la procédure pénale. 


\section{L'instruction}

L'instruction se déroule sous le contrôle de la police qui prépare un dossier contenant l'ensemble des preuves à charge. En droit français on pourrait la comparer à une enquête de police judiciaire.

Seront uniquement pris en compte les faits relatifs du dossier, l'instruction n'ayant pas à connaître de la personnalité du suspect. Seront ainsi collectés tous faits, indices corroborés le cas échéant par les dépositions des témoins, des rapports d'experts et tous autres éléments matériels. Les dépositions du prévenu, dès lors qu'il en exprime le désir ses déclarations, seront également consignées dans le dossier d'instruction.

\section{Le renvoi à l'audience}

Sous réserve qu'une mise en accusation ait été prononcée par un "Magistrate", le dossier est ensuite transmis au procureur qui n'est pas tenu par la decision du Procureur de telle sorte qu'il peut soit s'en tenir à la qualification retenue par le Magistrate soit en préférer une autre, soit décider qu'il n'y a pas lieu à poursuite.

\section{Le procès}

Ici, la procédure est accusatoire en ce sens que le procureur à seul la charge d'établir la preuve de la culpabilité de l'accusé. Pour ce faire, il se fondera sur un ensemble de preuves qu'il portera à la connaissance du jury et également de l'accusé ou de son conseil. Ce dernier devant à l'inverse, faire valoir devant ce même jury tous les éléments tendant à établir l'absence de culpabilité de son client. Le tribunal, c'est-à-dire, le juge et le jury, portant uniquement son attention sur les fait ayant un rapport avec le crime ou le délit. En conséquence la cour n'est pas autorisée à connaître le casier judiciaire de l'accusé, ainsi que tous éléments de la personnalité de l'accusé qui pourraient lui préjudicier. A l'inverse de la conception française, "on juge les faits et non pas l'homme".

\section{Le processus d'élaboration de la peine et son prononcé (les pays de Common Law parlent de "sentencing")}

Il s'agit d'un rôle dévolu au juge seul, sous réserve bien entendu que le jury ait préalablement déclaré l'accusé coupable. Avant le prononcé du jugement, la cour prend alors connaissance du casier judiciaire de l'accusé ainsi que de l'ensemble des éléments relatifs à sa personnalité. L'avocat de l'accusé pouvant le cas échéant faire entendre tous témoins de moralité qu'il souhaite et voire plaider sur la peine. On notera que le Procureur peut, bien qu'il use rarement de cette possibilité, fournir des explications orales. En Australie, c'est la deuxième étape du processus, qui revêt le plus d'importance. C'est en effet à cette stade que le destin de l'accusé est scellé. Par certains aspects les procès pénaux s'apparentent à une compétition sportive entre le Procureur et la défense, le plus pugnace et le mieux préparé l'emporte souvent. 


\section{L'appel}

La personne condamnée peut relever appel contre sa condamnation ou sa peine, ou les deux. Le procureur peut également relever appel mais seulement s'il estime la peine insuffisante. Un premier appel est possible auprès d'une Court of Criminal Appeal d'un État particulier, puis le cas échéant une deuxième fois devant la High Court of Australia sous réserve d'avoir obtenu l'autorisation préalable de cette derniere.

Le système pénal australien dans lequel le procès demeure le point culminant, ne présente le même caractère de continuité qu'on lui connaît en droit français dans lequel l'accent est plutôt mis sur la réunion des preuves tendant à établir la culpabilité du prévenu.

\section{Section II: Caractéristiques majeures de chaque étape de la procédure pénale australienne}

\section{§1-L'instruction}

Elle est du ressort quasi exclusif de la police. En effet hormis pour la délivrance des mandats de perquisition et, sous certaines conditions, des mandats d'amener, les membres de la police ne dépendent pas du juge d'instruction, tout comme ils ne sont pas sous le contrôle des procureurs. Dés lors, en pratique, et a priori , tout ce que peut souhaiter le prévenu (ce qui peut se révéler bien aléatoire car il n'y a pas de vraie garantie formelle offerte au prévenu), c'est que la police veuille bien s'en tenir au respect des dispositions légales et principes généraux du droit pénal applicables dans le cadre de leurs enquêtes. En fait le contrôle de la légalité de l'enquête, est opéré $a$ posteriori par les juges qui lors du procès pourront rejeter toutes preuves obtenues au mépris des règles légales ou déontologiques. Ainsi le juge opère un contrôle $a$ posteriori.

Les interrogatoires du prévenu sont évidemment du seul ressort de la police. On relèvera que si le prévenu conserve durant la phase d'instruction un droit fondamental au silence, ce privilège vaut aussi lors du procès. Les interrogatoires doivent respecter le principe selon lequel si les membres de la police sont autorisés à poser les questions que bon leur semble, ils doivent impérativement néanmoins, à partir du moment où des soupçons apparaissent, indiquer au prévenu que rien ne l'oblige à répondre à leurs questions. A défaut, l'ensemble des déclarations recueillies ne pourront pas etre retenues par la cour.

La question des aveux des prévenus, suscite de nombreuses controverses tant avant que pendant les procès. Cela est surtout vrai pour les aveux qui ne sont corroborés par aucun écrit, ou encore lorsque l'aveu écrit n'est pas signé. Un haut magistrat de la "High Court" (La Cour Suprême en Australie) a déclaré que les memibres du jury devaient être avertis des risques qui s'attachent à une condamnation prise sur le seul fondement d'un aveu de l'accusé, aveu ultérieurement contesté et non corroboré par des preuves matérielles 
indubitables. Il reste toutefois que cela est loin d'etre la règle aujourd'hui de telle sorte que le jury, ne dispose que de peu d'information à cet égard lors des procès .

Pour tenter de palier à d'éventuelles contestations, on a maintenant recours aux procédés offerts par les progrès de la technologie; ainsi il est possible de procéder à l'enregistrement vidéo des interrogatoires des suspects, comme cela est déjà le cas depuis quelques années en Nouvelle Galles du Sud, dans le cadre des interrogatoires menés par la "Drug Crime Commission" (la Commission contre les infractions à la législation des stupéfiants). Il en va plus récemment de même pour les interrogatoires ayant eu lieu dans les postes de police pour tous crimes ou délits d'importance. A ce jour, les réticences dues aux anciennes habitudes vaincues et les inévitables problèmes budgétaires réglés, les services de police ont dans leur ensemble fini par accepter d'adopter ce procédé, de telle sorte qu'en principe seuls les aveux enregistrés par vidéo pourront etre produits en justice. On attend néanmoins toujours un cadre législatif et réglementaire qui assure les meilleurs garanties légales à ce système.

L'assistance d'un conseil n'est pas garanti de manière dans l'ensemble des etats de l'Australie et ce malgré une recommandation formelle de la Law reform Commission. Ainsi si ce droit est maintenant reconnu en dans l'Etat de Victoria et dans le cadre de toutes procédures fédérales, il n'en va pas de même dans l'Etat de Nouvelle Galles du Sud.

\section{§ 2-La décision de renvoi à l'audience}

Une fois l'instruction terminée et avant que le prévenu ne puisse être renvoyé en procès devant un jury, une condition préalable doit cependant etre remplie. Il convient de satisfaire à la procédure dite "Committee Proceeding", à savoir une procédure de la mise en accusation d'un prévenu. Elle se déroule devant un "Magistrate" (un juge d'un cour inférieure mais spécialement investi d'un pouvoir exécutif particulier). A l'instigation du procureur (auparavant ce rôle de présentation était dévolu à un policier ayant reçu une formation spéciale à cet effet), tous les témoins à charge devront alors présentés et publiquement entendus par un "Magistrate", un contre-interrogatoire ("Cross-examination") étant en tant que de besoin, laissé à la discrétion de l'avocat de la défense. De surcroît, bien que cela soit assez rare dans la pratique, la défense peut elle aussi, user de son droit de faire entendre ses propres témoins.

Sur le fondement de ces témoignages (qui peuvent également inclure les aveux de l'inculpé recueillis par la police), le "Magistrate" doit décider s'il y a un "prima facie case" contre le prévenu, c'est-à-dire un ensemble de preuves étayées pouvant conduire à la condamnation par un jury du prévenu. Dans l'affirmative, il renvoie le prévenu devant une cour supérieure. dans la négative, il prononce la libération du prévenu. Statistiquement, on constate que pratiquement toujours les "Magistrates" se prononcent pour le renvoi du prévenu devant une cour supérieure, ce qui fait dire que cette procédure n'a guère de valeur. Mais, dès lors 
que le caractère accusatoire de la procédure interdit aux avocats de la défense d'avoir accès au dossier de la police ou du procureur, les premiers veulent néanmoins conserver la procédure dite "Committee Proceeding", en faisant valoir qu'elle permet au moins d'avoir connaissance des preuves dont dispose l'accusation, preuves sans lesquelles il est difficile de se ménager des arguments de défense cohérents.

Néanmoins, s'il n'entre pas dans les intentions de la défense de procéder à un contreinterrogatoire des témoins de l'accusation, on se contentera alors d'une simple référence de leurs dépositions écrites, appelé "Paper Commital". Nonobstant la décision de renvoi devant un cour supérieure par le "Magistrate" prise à l'encontre du prévenu, le Procureur Général ("The Director of Public Prosecutions") conserve toujours le pouvoir d'infirmer cette décision. Toutefois, dans la pratique, il n'intervient guère, la décision de renvoi du "Magistrate" étant simplement implicitement confirmée.

Le gouvernement en Nouvelle Galles du Sud a comme projet propose d'attribuer au Procureur Général et à lui seul, le pouvoir de procéder au renvoi du prévenu devant la juridiction répressive un prévenu. Ce projet n'est pas sans susciter d'importantes controverses, car l'exclusion du "Magistrate" a aussi pour conséquence le passage d'une procédure publique à une procédure secrète.

\section{§ 3- Le Procès Pénal devant les Cours Supérieures}

\section{A Les parties au procès pénal}

\section{Le juge}

Les juges australiens, sont recrutés parmi les avocats les plus chevronnés et appointés par le gouvernement. Ils n'ont pas reçu de formation spécifique pour ces fonctions particulières, si ce n'est à travers leur précédente expérience personnelle. Le juge préside le procès pénal. Toutes les questions de droit sont tranchées par lui, en particulier ce qui doit etre admis ou rejette comme preuve devant le jury.

Avant que le jury ne se retire pour délibérer sur la culpabilité du prévenu, il incombe au juge dans le cadre d'un "summing up", de porter à la connaissance des membres du jury et de lui expliquer l'ensemble des règles de droit pénal applicables à l'affaire qui leur est soumise, les éléments constitutifs des faits reprochés à l'accusé, ainsi que ceux pouvant établir son innocence. Le juge résume ainsi au jury les points fondamentaux des témoignages qui leurs ont été soumis et tout ce qui leur permettra de se prononcer. $\mathrm{Si}$, le jury après délibération déclare le prévenu coupable, il appartiendra au juge seul de fixer sa peine. 


\section{Le jury}

Il est constitué de douze membres, personnes physiques, choisies par tirage au sort sur une liste électorale. La défense et le procureur pouvant par trois fois (en Nouvelle Galles du Sud), à tour de rôle, user de leur droit de récusation.

Le jury se prononce sur toutes les questions de fait, et in fine sur la culpabilité ("guilty") ou non de l'accusé ("not guilty"). Dés lors qu'existe un doute raisonnable sur la culpabilité de l'accusé, le jury peut se prononcer pour un acquittement. Quelque soit la décision retenue (culpabilité ou acquittement) la décision du jury doit toujours être unanime ( au moins en Nouvelle Galles du Sud), à défaut un nouveau procès doit normalement avoir lieu.

\section{Le Procureur}

Les procureurs sont nommés par le gouvernement (en fait par le Garde des Sceaux) parmi les avocats en exercice. Une fois nommés, si leurs activités doivent se limiter à celles dévolues à tous membres du Parquet, ils conservent néanmoins certaines prérogatives de leurs anciennes fonctions d'avocat, c'est-à-dire une totale indépendance dans leur manière de préparer le procès dont ils ont la charge. Les procureurs représentent "the Crown" (la Couronne), c'est-à-dire l'Etat dans les procès pénaux. C'est le procureur qui supporte la charge de la preuve de la culpabilité de l'accusé devant la cour. Pour se faire, il expose en premier lieu l'ensemble des faits au jury puis, il mène l'interrogatoire des témoins qu'il souhaite faire entendre, tout comme celui des témoins delà défense, dans le cadre du respect du principe du contradictoire. Enfin, devant le jury, il prend toutes les réquisitions, qui lui semblent appropriées quant à la culpabilité du prévenu ou de l'accusé.

\section{L'Avocat de la Défense}

Il est soit librement choisi par l'accusé ou bien nommé au titre de l'assistance judiciaire. Dans ce dernier cas le conseil, tout comme un procureur, est nommé par le gouvernement parmi les avocats en exercice et perçoit le même traitement qu'un procureur. L'avocat de la défense interroge de manière contradictoire l'ensemble des témoins entendus par le procureur et le cas échéant produit à la barre les témoins de la défense. Il plaide après le procureur et tout comme lui, il base son argumentation sur les preuves présentées à la cour et portant uniquement sur la question de la culpabilité. Plus tard, si son client et reconnu coupable, il plaidera à nouveau sur le bien fondé et le quantum de la peine à infliger.

\section{L'accusé}

Il a droit à bénéficier de l'assistance d'un conseil durant tout son procès et ce y compris dans sa phase préparatoire.

Trois options s'ouvrent à lui dans le cadre des explications qu'il sera amené à fournir à la cour. Il peut tout d'abord décider de prendre la qualité de témoin, dès lors il prêtera 
serment et partant il encoure le risque de subir un interrogatoire contradictoire de la part du Procureur. Il peut ensuite, faire un "statement from the dock", c'est-à-dire une déclaration du banc des accusés, sans avoir à prêter serment et en évitant ainsi un interrogatoire du Procureur. Enfin, en troisième lieu, il peut garder le silence tout le long du procès. Dans cette derniere hypothèse, ni le juge ni le procureur n'ont le droit d'en tirer des conséquences pour le jury.

\section{B Les Règles de Preuves}

C'est une partie importante du droit pénal australien. On a souvent fait valoir que ces règles ont été élaborées afin de conférer au jury, une protection contre la faiblesse des preuves qui viendraient à lui etre soumises. Ainsi pour la plupart ces règles tendent à l'exclusion de certaines preuves. En règle générale, et à titre d'exemple, ne seront pas admissibles: les preuves par ouï-dire (c'est-à-dire, ce qu'un autre a dit au témoin au sujet des fait en litige), les preuves fondées sur l'opinion du témoin s'il n'est pas un expert sur le sujet, les preuves sur un aspect négatif de la personnalité de l'accusé ("Bad Character"). Sous réserve que l'une des parties conteste durant le procès, la recevabilité d'une preuve particulière, c'est au juge seul qu'il appartient d'en décider. Ainsi, à défaut de contestation, une preuve même attaquable sur le principe, sera retenue.

Il reste néanmoins que le juge dispose toujours d'un droit discrétionnaire pour refuser toutes preuves qu'il n'estime pas appropriées au bon déroulement du procès.

Par exemple, dans le cas d'aveux recueillis par à la police, les règles de droit applicables postulent que l'aveu doit être volontaire (c'est-à-dire en respectant la possibilité ouverte au prévenu de se taire). Mais quand bien même cela aurait été le cas, si le juge relève qu'il serait injuste dans les circonstances de l'admettre (par exemple si l'accusé était blessé ou voire trop fatigué), ou s'il constate que l'aveu a été obtenu de manière illégale (par exemple, après une détention trop longue), il peut toujours l'écarter de sa propre initiative.

Au moment du "summing-up", le juge rappellera au jury, que leur décision devra uniquement être basée sur les seules preuves retenues au cours de l'audience. Si le juge prend un certain nombre de notes sur les témoignages qui ont été entendus, il se référera surtout à la transcription en clair du sténogramme de tous les témoignages du procès. Ce document est mis un jour après sa transcription, à la disposition du juge, du procureur et de l'accusé et de son avocat. Il en va ainsi tout au long du procès. Ces transcriptions servent de base au procureur et à l'avocat de la défense dans le cadre des interrogatoires contradictoires des témoins ainsi que pour leurs plaidoiries. Le jury, toutefois, n'aura pas accès à cette transcription, de telle sorte que les jurés ne pourront se fier qu'à leurs propres notes et leur mémoire. 


\section{§ 3-L' appel}

Droit ouvert tant à l'accusé et qu'au procureur (c'est-à-dire "the Crown"- la Couronne), l'appel est porté dans l'Etat de Nouvelle Galles du Sud devant la "Court of Criminal Appeal" (La Cour d'Appel en matière pénale, le même système prévalant dans les autres Etats d'Australie). Trois juges la compose avec à leur tète, un président.

L'accusé peut relever appel sur toutes les questions de droit révélées dans le cadre du procès à savoir tout ce qui relève de l'admissibilité des preuves à l'audience, des explications fournies par le juge au jury. Est également possible l'appel contre les décisions du jury qui doivent reposer sur les seuls témoignages et faits matériels apparus au cours du procès.

L'appel ayant été jugé recevable en la forme, la Cour d'Appel prononcer l'acquittement, la confirmation de la decision de première instance (tel peut etre le cas même si des irrégularités ont été constatées sous réserve cependant qu'il n'y ait pas eu de déni de justice), ou encore ordonner un nouveau procès. L'accusé peut aussi limiter son appel au seul quantum de la peine et chercher en conséquence à la réduire, l'appréciation de la Cour d'Appel étant à cet égard souveraine.

Le procureur quant à lui, voit son pouvoir de faire appel limité aux seules questions de droit. En tout état de cause, son appel ne peut jamais avoir pour effet de remettre en cause une décision préalable d'acquittement prise par un jury dont la décision doit demeurer intangible. Le procureur peut aussi relever appel contre l'insuffisance de la peine infligée en première instance.

Chaque fois que se pose une question de droit de portée générale, une deuxième procédure d'appel pourra alors etre engagée devant la "High Court" (la Cour Suprême d'Australie), il reste assez rare en pratique et de plus il est subordonné à une autorisation préalable de cette même juridiction.

\section{§ 4- Aveu de culpabilité fait à l'audience (Plea of guilty)}

Les explications précédentes portaient sur le déroulement d'un procès au cours duquel l'accusé clame son innocence et conteste des faits qui lui étaient reprochés. Dans ce cas-là il appartient au procureur de prouver "au-delà de tout doute raisonnable" que l'accusé est véritablement coupable. A défaut l'accusé sera acquitté. Mais, il reste bien sur, la situation où sur les conseils de son avocat et sur la foi de la transcription des témoignages recueillis devant le "Magistrate" au moment du "Committal Proceeding", que l'accusé accepte de reconnaître à l'audience les faits qui lui sont reprochés. Si tel devait etre le cas, des renseignements complémentaires sur les faits étant donnés par le procureur, le juge décide alors s'il peut accepter ou non l'aveu de l'accusé. Dans l'affirmative, il considère alors, tout de suite ou après une remise de séance, de la peine à infliger. Dès lors qu'un aveu a été 
valablement accepté, il n'y a plus lieu d'avoir recours aux services du jury ainsi qu'à l'audition des témoins à charge. Il reste toujours cependant loisible à l'accusé de faire entendre ses propres témoins, pour tenter de minorer le quantum de la peine qui sera prononcée. A ceci s'ajoute la possibilité pour le juge de se référer à toutes expertises psychiatriques et psychologiques qu'il jugera utiles.

\section{§ 5-La publicité et les procès devant les juridictions pénales}

Le principe de la liberté de la presse est un des fondements les plus importants dans une démocratie. On en déduit qu'un accusé a le droit d'être exclusivement jugé par une cour, comprenant le cas échéant un jury. Sur ce fondement, les juridictions australiennes interdisent la publication, par voies de presse qu'elle soit écrite, radiophonique, ou encore audiovisuelle, de tout qui pourrait etre de nature à influencer une juridiction, et surtout un jury. La transgression de cette interdiction constitue un outrage à magistrat passible de peine d'amende ou de prison. La presse peut cependant toujours se faire l'écho des procès et les instructions sous réserve que les informations publiées soient exactes. En Australie, il y a une nette tendance pour la police de faire de la publicité relative aux arrestations et leurs enquêtes en cours, et il n'est pas rare que des conférences de presse soient aussi organisées. Il y a là un dérapage contestable qui peut en certaines circonstances peut réellement s'analyser en un outrage à magistrats.

\section{Overview of the Australian law of criminal procedure}

The Australian Federal Constitution of 1900 distributes legislative powers between the Federal Parliament of the Commonwealth of Australia (sitting in Canberra) and the parliaments of the six states of Australia. Criminal law, except in respect of those matters which are within its federal jurisdiction, is outside the legislative domain of the Federal Parliament, and therefore only the states can legislate in this area. The criminal law of three states of Australia (New South Wales, Victoria, and South Australia) appears as a mixture of legislation and the common law. Three other states (Queensland, Western Australia, and Tasmania) have criminal codes which date from the beginning of the 20th century.

In Australia, criminal process depends on the form of trial: with jury or without jury. Schematically, trial with jury is reserved for those offences which could be described as serious (eg assassinations, murders, rapes, assaults with grievous bodily harm, and robbery).

There are three main steps to be considered in the conduct of a criminal trial: first, there is the investigation which is under the almost exclusive control of the police; the courts have a control which is a posteriori; then there is the trial; and the third step is sentencing. 
Australian judges are recruited from among the most experienced barristers, and appointed by the government. They receive no specific training for their judicial functions. The judge presides over the criminal trial. All decisions of law are decided by the judge, and in particular what matters of evidence may be put before the jury. The judge sums up for the jury the main points of the evidence given at the trial and advises the jury about all that they have to decide. If the jury after deliberation returns a verdict of guilty, the judge alone will decide on the punishment.

The jury is of twelve persons and decides all matters of fact and, ultimately, the culpability or otherwise of the accused. Whatever the decision of the jury, it must be unanimous. If the jury is not agreed a new trial will normally take place.

Prosecutors are appointed by the government (ie by the Attorney-General) from among practising barristers. They represent the Crown, that is to say the state, in criminal trials and they have the burden of proof of the guilt of accused.

The defence lawyer is chosen freely by the accused or appointed by the court by way of legal aid.

The rules of evidence are an important part of Australian criminal law. For the most part, these rules are concerned with the exclusion of certain types of evidence. The judge however always has a discretion to refuse to admit any evidence which he or she believes is inappropriate to the proper conduct of the trial.

Appeal is available both to the accused and to the prosecutor, that is to say, the Crown. Appeals are heard by a criminal division of a Court of Appeal.

Publicity in respect of the criminal trials presents a problem that is difficult to resolve. In principle, the Australian courts prohibit the publication by the media, whether in written, radio or audio-visual form of any matter which could influence a court or a jury. A breach of this prohibition amounts to a contempt of court which is punishable by fine or a term of imprisonment. 